\title{
VARIOUS SHADOWING PROPERTIES AND THEIR EQUIVALENT FOR EXPANSIVE ITERATED FUNCTION SYSTEMS
}

\author{
MEHDI FATEHI NIA
}

Received 08 April, 2015

\begin{abstract}
In this paper we introduce expansive iterated function systems, (IFS) on a compact metric space then various shadowing properties and their equivalence are considered for expansive IFS.

2010 Mathematics Subject Classification: 37C50; 37C15
\end{abstract}

Keywords: eExpansive IFS, pseudo orbit, shadowing, continuous shadowing, limit shadowing, Lipschitz shadowing

\section{INTRODUCTION}

The notion of shadowing plays an important role in dynamical systems, specially; in stability theory $[1,11,12]$. Various shadowing properties for expansive maps and their equivalence have been studied by Lee and Sakai [10,14]. More precisely, they prove the following theorems:

Theorem 1 ([14]). Let $f$ be an expansive homeomorphism on a compact metric space $(X ; d)$. Then the following conditions are mutually equivalent:

(a) $f$ has the shadowing property,

(b) $f$ has the continuous shadowing property,

(c) there is a compatible metric D for $X$ such that $f$ has the Lipschitz shadowing property with respect to $D$,

(d) $f$ has the limit shadowing property,

(e) there is a compatible metric $D$ for $X$ such that $f$ has the strong shadowing property with respect to $D$.

Theorem 2 ([10]). Let $f$ be a positively expansive map on a compact metrizable space $X$. Then the following conditions are mutually equivalent:

(a) $f$ is an open map,

(b) $f$ has the shadowing property,

(c) there is a metric such that $f$ has the Lipschitz shadowing property,

(d) there is a metric such that $f$ has the s-limit shadowing property,

(e) there is a metric such that $f$ has the strong shadowing property. 
In the other hand, iterated function systems( IFS), are used for the construction of deterministic fractals and have found numerous applications, in particular to image compression and image processing [2]. Important notions in dynamics like attractors, minimality, transitivity, and shadowing can be extended to IFS (see [3, 4, 7-9]). The authors defined the shadowing property for a parameterized iterated function system and prove that if a parameterized IFS is uniformly expanding ( or contracting), then it has the shadowing property[9].

In this paper we present an approach to shadowing property for iterated function systems. At first, we introduce expansive iterated function systems on a compact metric space. Then continuous shadowing, limit shadowing and Lipschitz shadowing properties are defined for an IFS, $\mathscr{F}=\left\{X ; f_{\lambda} \mid \lambda \in \Lambda\right\}$ where $\Lambda$ is a nonempty finite set and $f_{\lambda}: X \rightarrow X$ is homeomorphism, for all $\lambda \in \Lambda$. Theorems 3 and 4 are the main result of the present work. Actually in these theorems we prove that the limit shadowing property, the Lipschitz shadowing property are all equivalent to the shadowing property for expansive IFS on a compact metric space. The method is essentially the same as that used in $[10,13,14]$. Finally, we introduce the strong expansive IFS and show that for a strong expansive IFS the continuous shadowing property and the shadowing property are equivalent.

\section{PRELIMINARIES}

In this section, we give some definitions and notations as well as some preliminary results that are needed in the sequel. Let $(X, d)$ be a complete metric space. Let us recall that an Iterated Function System(IFS)

$\mathscr{F}=\left\{X ; f_{\lambda} \mid \lambda \in \Lambda\right\}$ is any family of continuous mappings $f_{\lambda}: X \rightarrow X, \lambda \in \Lambda$, where $\Lambda$ is a finite nonempty set (see[9]).

Let $\Lambda^{\mathbb{Z}}$ denote the set of all infinite sequences $\left\{\lambda_{i}\right\}_{i \in \mathbb{Z}}$ of symbols belonging to $\Lambda$. A typical element of $\Lambda^{\mathbb{Z}}$ can be denoted as $\sigma=\left\{\ldots, \lambda_{-1}, \lambda_{0}, \lambda_{1}, \ldots\right\}$ and we use the shorted notation

$$
\begin{gathered}
\mathcal{F}_{\sigma_{0}}=i d, \\
\mathcal{F}_{\sigma_{n}}=f_{\lambda_{n-1}} o f_{\lambda_{n-2}} o \ldots o f_{\lambda_{0}}, \\
\mathcal{F}_{\sigma_{-n}}=f_{\lambda_{-n}}^{-1} o f_{\lambda_{-(n-1)}}^{-1} o \ldots o f_{\lambda_{-1}}^{-1} .
\end{gathered}
$$

Please note that if $f_{\lambda}$ is a homeomorphism map for all $\lambda \in \Lambda$, then for every $n \in \mathbb{Z}$ and $\sigma \in \Lambda^{\mathbb{Z}}, \mathcal{F}_{\sigma_{n}}$ is a homeomorphism map on $X$.

A sequence $\left\{x_{n}\right\}_{n \in \mathbb{Z}}$ in $X$ is called an orbit of the IFS $\mathscr{F}$ if there exist $\sigma \in \Lambda^{\mathbb{Z}}$ such that $x_{n+1}=f_{\lambda_{n}}\left(x_{n}\right)$, for each $\lambda_{n} \in \sigma$.

The IFS $\mathscr{F}=\left\{X ; f_{\lambda} \mid \lambda \in \Lambda\right\}$ is uniformly expanding if there exists

$$
\beta=\inf _{\lambda \in \Lambda} \inf _{x \neq y} \frac{d\left(f_{\lambda}(x), f_{\lambda}(y)\right)}{d(x, y)}
$$


and this number called also the expanding ratio, is greater than one [9].

We say that $\mathscr{F}$ is expansive if there exist a $e>0$ such that for every arbitrary $\sigma \in \Lambda^{\mathbb{Z}}$, $d\left(\mathcal{F}_{\sigma_{i}}(x), \mathcal{F}_{\sigma_{i}}(y)\right)<e$, for all $i \in \mathbb{Z}$, implies that $x=y$.

Remark 1. Let $\mathcal{F}$ be an uniformly expanding IFS and $\beta>1$ is it's expanding ratio number. Suppose that for every $\sigma \in \Lambda^{\mathbb{Z}}, \mathcal{F}_{\sigma_{n}}$ and $z \in X,\left\{\mathcal{F}_{\sigma_{n}}(z)\right\}_{n \in Z}$, is an infinite set.

Consider the number $1<\gamma<\beta$, since $\Lambda$ is a finite set there exists at most a finite set of points $x, y \in X$ such that $\frac{d\left(f_{\lambda}(x), f_{\lambda}(y)\right)}{d(x, y)} \leq \gamma$, for some $\lambda \in \Lambda$. Now, Let $\sigma=\left\{\ldots, \lambda_{-1}, \lambda_{0}, \lambda_{1}, \ldots\right\}$ be an element of $\Lambda^{\mathbb{Z}}$ and consider distinct points $x, y \in$ $X$. Suppose that $d\left(\mathscr{F}_{\sigma_{i}}(x), \mathscr{F}_{\sigma_{i}}(y)\right)<1$ for all $i \in \mathbb{Z}$. Since every orbit is an infinite set and $f_{\lambda_{i}}$ is injective, for all $i \in Z$, then there exists $k>0$ such that $\frac{d\left(f_{\lambda_{k+i}}\left(\mathcal{F}_{\sigma_{k+i-1}}(x)\right), f_{\lambda_{k+i}}\left(\mathcal{F}_{\sigma_{k+i-1}}(y)\right)\right)}{d\left(\mathscr{F}_{\sigma_{k+i-1}}(x), \mathcal{F}_{\sigma_{k+i-1}}(y)\right)}>\gamma$, for all $i \geq 0$. So, $\left.\gamma^{i} d\left(\mathcal{F}_{\sigma_{k}}(x)\right), \mathcal{F}_{\sigma_{k}}(y)\right)$ $<d\left(\mathcal{F}_{\sigma_{k+i}}(x), \widetilde{F}_{\sigma_{k+i}}(y)\right)<1$, for all $i>0$, and consequently $x=y$. Then uniformly expanding implies the expansivity.

Also following example shows that expansivity does not imply uniformly expanding.

Example 1. Let $\Sigma$ denote the set of all bi-infinite sequence $x=\left(\cdots, x_{-2}, x_{-1}, x_{0}, x_{1}, x_{2}, \cdots\right)$ where $x_{n}=0$ or 1 . The set $\Sigma$ becomes a compact metric space if we define the distance between two points $x, y$ by $\rho(x, y)=$ $\sum_{i=-\infty}^{\infty} \frac{\left|x_{i}-y_{i}\right|}{2^{|i|}}$. This is well known that he shift map $g: \Sigma \longrightarrow \Sigma$ defined by $(\sigma(x))_{i}=x_{i+1}$ is a homeomorphism and so by compactness of $\Sigma$ is not uniformly expanding. If $x \neq y$ are two point in $\Sigma$ then, for some $k \in Z, x_{k} \neq y_{k}$ and hence $\rho\left(g^{k}(x), g^{k}(y)\right) \geq 1$, this implies that $g: \Sigma \longrightarrow \Sigma$ is an expansive homeomorphism. Let $f: \Sigma \longrightarrow \Sigma$ be a map defined by $(f(x))_{i}=x_{i+2}$, using the above arguments, we can prove that if $x \neq y$ are two point in $\Sigma$ then, for some $k \in Z, \rho\left(f^{k}(x), f^{k}(y)\right) \geq$ $\frac{1}{2}$. Now consider the IFS $\mathcal{F}=\left\{\Sigma ; f_{i} \mid i \in\{1,2\}\right\}$, where $f_{1}=g$ and $f_{2}=f$. If $x \neq y$ are two point in $\Sigma$ then, for every $\sigma \in \Lambda^{Z}$, there exists $k \in Z$ such that $\rho\left(\widetilde{F}_{\sigma_{k}}(x), \widetilde{F}_{\sigma_{k}}(y)\right) \geq \frac{1}{2}$. So the IFS $\mathcal{F}=\left\{\Sigma ; f_{i} \mid i \in\{1,2\}\right\}$ is an expansive IFS contains homeomorphism functions and is not uniformly expanding.

Given $\delta>0$, a sequence $\left\{x_{i}\right\}_{i \in \mathbb{Z}}$ in $X$ is called a $\delta$-pseudo orbit of $\mathscr{F}$ if there exist $\sigma \in \Lambda^{\mathbb{Z}}$ such that for every $\lambda_{i} \in \sigma$, we have $d\left(x_{i+1}, f_{\lambda_{i}}\left(x_{i}\right)\right)<\delta$.

One says that the IFS $\mathcal{F}$ has the shadowing property if, given $\epsilon>0$, there exists $\delta>0$ such that for any $\delta$-pseudo orbit $\left\{x_{i}\right\}_{i \in \mathbb{Z}}$ there exist an orbit $\left\{y_{i}\right\}_{i \in \mathbb{Z}}$, satisfying the inequality $d\left(x_{i}, y_{i}\right) \leq \epsilon$ for all $i \in \mathbb{Z}$. In this case one says that the $\left\{y_{i}\right\}_{i \in \mathbb{Z}}$ or the point $y_{0}, \epsilon-$ shadows the $\delta$-pseudo orbit $\left\{x_{i}\right\}_{i \in \mathbb{Z}}[9]$.

Please note that if $\Lambda$ is a set with one member then the IFS $\mathcal{F}$ is an ordinary discrete dynamical system. In this case the shadowing property for $\mathcal{F}$ is ordinary shadowing 
property for a discrete dynamical system.

The point $y_{0}$ in definition of shadowing property related to the sequence $\sigma$ which makes the orbit $\left\{y_{i}\right\}_{i \in \mathbb{Z}}$ is unique.

Remark 2. Suppose that the IFS $\mathcal{F}$ satisfied shadowing property and is expansive with expansive constant $e>0$. Consider $0<\epsilon<\frac{e}{3}$ and choose $\delta>0$ corresponding to $\epsilon$ in the definition of shadowing property. Let $\left\{x_{i}\right\}_{i \in \mathbb{Z}}$ be a $\delta$-pseudo orbit for IFS, there exist a sequences $\sigma=\left\{\ldots, \mu_{-1}, \mu_{0}, \mu_{1}, \ldots\right\}$ and $\left\{y_{i}\right\}_{i \in \mathbb{Z}}$ such that $y_{i+1}=$ $f_{\mu_{i}}\left(y_{i}\right)$ and $d\left(x_{i}, y_{i}\right)<\epsilon$, for all $i \in \mathbb{Z}$. If $\left\{z_{i}\right\}_{i \in \mathbb{Z}}$ be another sequence such that $z_{i+1}=f_{\mu_{i}}\left(z_{i}\right)$ and $d\left(x_{i}, z_{i}\right)<\epsilon$, for all $i \in \mathbb{Z}$,

then for all $i \in \mathbb{Z}$ :

$$
d\left(\mathcal{F}_{\sigma_{i}}\left(y_{0}\right), \mathcal{F}_{\sigma_{i}}\left(z_{0}\right)\right)=d\left(y_{i}, z_{i}\right) \leq d\left(y_{i}, x_{i}\right)+d\left(x_{i}, z_{i}\right)<2 \epsilon<e .
$$

Thus by expansivity of the IFS $\mathcal{F}$ we have that $y_{0}=z_{0}$.

We say that $\mathcal{F}$ has the Lipschitz shadowing property if there are $L>0$ and $\epsilon_{0}>0$ such that for any $0<\epsilon<\epsilon_{0}$ and any $\epsilon$-pseudo orbit $\left\{x_{i}\right\}_{i \in \mathbb{Z}}$ of $\mathcal{F}$ there exist $y \in X$ and $\sigma \in \Lambda^{\mathbb{Z}}$ such that $d\left(\mathcal{F}_{\sigma_{i}}(y), x_{i}\right)<L \epsilon$, for all $i \in \mathbb{Z}$.

We say that $\mathcal{F}$ has the limit shadowing property if: for any sequence $\left\{x_{i}\right\}_{i \in \mathbb{Z}}$ of points in $X$, if $\lim _{i \rightarrow \pm \infty} d\left(f_{\lambda_{i}}\left(x_{i}\right), x_{i+1}\right)=0$, for some

$\sigma=\left\{\ldots, \lambda_{-1}, \lambda_{0}, \lambda_{1}, \ldots\right\} \in \Lambda^{\mathbb{Z}}$ then there is an orbit $\left\{y_{i}\right\}_{i \in \mathbb{Z}}$ such that $\lim _{i \rightarrow \pm \infty} d\left(x_{i}, y_{i}\right)=0$

Let $X^{Z}$ be the set of all sequences $\left\{x_{i}\right\}_{i \in \mathbb{Z}}$ of points in $X$ and let $\widetilde{d}$ be the metric on $X^{Z}$ defined by

$$
\widetilde{d}\left(\left\{x_{i}\right\}_{i \in \mathbb{Z}} ;\left\{y_{i}\right\}_{i \in \mathbb{Z}}\right)=\sup _{i \in \mathbb{Z}} \frac{d\left(x_{i} ; y_{i}\right)}{2^{|i|}},
$$

for $\left\{x_{i}\right\}_{i \in \mathbb{Z}},\left\{y_{i}\right\}_{i \in \mathbb{Z}} \in X^{Z}$. Let $p(\mathcal{F}, \delta)$ be the set of all $\delta$-pseudo-orbits $(\delta>0)$ of $\mathcal{F}$ with the subspace topology of $X^{Z}$ [14].

We say that $\mathcal{F}$ has the continuous shadowing property if for every $\epsilon>0$, there are a $\delta>0$ and a continuous map $r: p(\mathcal{F}, \delta) \rightarrow X$ such that

$d\left(\mathcal{F}_{\sigma_{i}}(r(\mathrm{x})), x_{i}\right)<\epsilon$, where $\sigma=\left\{\ldots, \lambda-1, \lambda_{0}, \lambda_{1}, \ldots\right\}, \mathrm{x}=\left\{x_{i}\right\}_{i \in \mathbb{Z}}$ and $d\left(f_{\lambda_{i}}\left(x_{i}\right), x_{i+1}\right)<\delta$, for all $i \in \mathbb{Z}$.

\section{RESUlts}

By Theorem 2, Sakai showed that any positively expansive open map has the shadowing property. In this section we introduce open IFS and show that for an expansive IFS, the openness; shadowing property and Lipschitz shadowing property are equivalent.

$\mathcal{F}=\left\{X ; f_{\lambda} \mid \lambda \in \Lambda\right\}$ is said to be an open IFS if $f_{\lambda}$ is an open map, for all $\lambda \in \Lambda$.

Definition 1 ([10]). Let $f: X \rightarrow X$ be a continuous map on a compact metric space. We say that $f$ expands small distances if there exist constants $\delta_{0}>0$ and 
$\alpha>1$ such that $0<d(x, y)<\delta_{0}(x, y \in X)$ implies

$d(f(x), f(y))>\alpha d(x, y)$.

We say that $\mathcal{F}$ expands small distance, if there are constants $\delta_{0}>0$ and $\alpha>1$ such that $d\left(f_{\lambda}(x), f_{\lambda}(y)\right)>\alpha d(x, y)$ whenever $0<d(x, y)<\delta_{0}(\lambda \in \Lambda)$

Remark 3. Suppose that $\mathscr{F}=\left\{X ; f_{\lambda} \mid \lambda \in \Lambda\right\}$ is an expansive IFS, then $f_{\lambda}$ is an expansive function and by Lemma 1 . of [10] expand small distance. Let in the proof of Lemma 1. of [10]

$V_{n}=\left\{(x, y) \in X \times X: d\left(\widetilde{F}_{\sigma_{i}}(x), \mathscr{F}_{\sigma_{i}}(y)\right) \leq c\right.$, for all $\sigma \in \Lambda^{\mathbb{Z}}$ and all $\left.|i|<n\right\}$.

So, $\mathcal{F}$ expand small distance.

To prove Theorem 3, we need the following lemma.

Lemma 1. Suppose that $\mathcal{F}$ expands small distance with related constants $\alpha>1$ and $\delta_{0}>0$, then the following are equivalent:

i) $\mathcal{F}$ is an open IFS.

ii) There exists $0<\delta_{1}<\frac{\delta_{0}}{2}$ such that if $d\left(f_{\lambda}(x), y\right)<\alpha \delta_{1}$ then $B_{\delta_{1}}(x) \cap f_{\lambda}^{-1}(y) \neq \varnothing$, for all $\lambda \in \Lambda$, where $B_{\delta_{1}}(x)$ is the neighborhood of $x$ with radius $\delta_{1}$.

Proof. Since every $f_{\lambda}$ expands small distance then by Lemma 2. of [10], for every $\lambda \in \Lambda$ the following are equivalent:

i) $f_{\lambda}$ is an open map.

ii ) there exists $0<\delta_{\lambda}<\frac{\delta_{0}}{2}$ such that if $d\left(f_{\lambda}(x), y\right)<\alpha \delta_{\lambda}$ then $B_{\delta_{\lambda}}(x) \cap f_{\lambda}^{-1}(y) \neq \varnothing$.

Because of the proof of Lemma 1 in [5] for every $\lambda \in \Lambda$ there exist infinitely $0<\delta<$ $\delta_{\lambda}$ such that $d\left(f_{\lambda}(x), y\right)<\alpha \delta$ implies $B_{\delta_{\lambda}}(x) \cap f_{\lambda}^{-1}(y) \neq \varnothing$. So this sufficient to take

$\delta_{1}=\min \left\{\delta_{\lambda}: \lambda \in \Lambda\right\}$.

Corollary 1. Let the IFS $\mathcal{F}=\left\{X ; f_{\lambda} \mid \lambda \in \Lambda\right\}$ expands small distance with related constants $\alpha>1$ and $\delta_{0}>0$, and satisfied condition $i i$ in Lemma 1. Then for all $0<\epsilon \leq \delta_{1}, \lambda \in \Lambda$ and $x, y \in X, d\left(f_{\lambda}(x), y\right)<\epsilon$ implies that $B_{\frac{\epsilon}{\alpha}}(x) \cap f_{\lambda}^{-1}(y) \neq \varnothing$.

Proof. Let $d\left(f_{\lambda}(x), y\right)<\epsilon$, since $\epsilon \leq \delta_{1}$ by Lemma 1 . we have that $B_{\delta_{1}}(x) \cap$ $f_{\lambda}^{-1}(y) \neq \varnothing$. Take $z \in B_{\delta_{1}}(x) \cap f_{\lambda}^{-1}(y)$. This is clear that $y=f_{\lambda}(z)$ and $d(x, z)<$ $\delta_{1}$. Since $\mathcal{F}$ expands small distances,

$$
\alpha d(x, z)<d\left(f_{\lambda}(x), f_{\lambda}(z)\right)=d\left(f_{\lambda}(x), y\right)<\epsilon .
$$

Thus $d(x, z)<\frac{\epsilon}{\alpha}$ and hence $B_{\frac{\epsilon}{\alpha}}(x) \cap f_{\lambda}^{-1}(y) \neq \varnothing$.

Theorem 3. Suppose that $\mathcal{F}$ expands small distance with related constants $\alpha>1$ and $\delta_{0}>0$, the following conditions are equivalent:

i) $\mathcal{F}$ is an open IFS. 
ii) $\mathcal{F}$ has the shadowing property.

i i i) $\mathcal{F}$ has the Lipschitz shadowing property.

Proof. ( $i$ i $\Rightarrow$ i $i$ ) By definitions of the shadowing and Lipschitz shadowing properties this is clear that the Lipschitz shadowing property implies the shadowing property.

( $i \Longrightarrow$ i i $i)$ Let $L=\frac{2 \alpha}{\alpha-1}=2 \Sigma_{k=0}^{\infty} \alpha^{-k}>1$ and fix any $0<\epsilon<\frac{\delta_{1}}{L}$, where $\delta_{1}$ and $\alpha$ be as in Lemma 1. Suppose that $\left\{x_{i}\right\}_{i \in \mathbb{Z}}$ is an $\epsilon-$ pseudo orbit for $\mathcal{F}$; there is $\sigma=\left\{\ldots, \lambda_{-1}, \lambda_{0}, \lambda_{1}, \ldots\right\} \in \Lambda^{\mathbb{Z}}$ such that $d\left(f_{\lambda_{i}}\left(x_{i}\right), x_{i+1}\right)<\epsilon$ for all $i \in \mathbb{Z}$.

Pick any $i \geq 1$ and put $\alpha_{j}=\Sigma_{k=0}^{j-1} \alpha^{-k}$ for $j \geq 1$. Since $d\left(f_{\lambda_{i}}\left(x_{i}\right), x_{i+1}\right)<\epsilon$ then, by Lemma 1 and Corollary 1 , there exists $y_{i-1}^{(i)} \in B_{\frac{\epsilon}{\alpha}}\left(x_{i-1}\right)$ such that $f_{\lambda_{i-1}}\left(y_{i-1}^{(i)}\right)=x_{i}$. Thus

$$
\begin{array}{r}
d\left(f_{\lambda_{i-2}}\left(x_{i-2}\right), y_{i-1}^{(i)}\right) \leq d\left(f_{\lambda_{i-2}}\left(x_{i-2}\right), x_{i-1}\right)+d\left(x_{i-1}, y_{i-1}^{(i)}\right) \\
<\epsilon+\frac{\epsilon}{\alpha}=\epsilon\left(1+\frac{1}{\alpha}\right)<\epsilon L .
\end{array}
$$

Hence there exists $y_{i-2}^{(i)} \in B_{\alpha_{2} \frac{\epsilon}{\alpha}}\left(x_{i-2}\right)$ such that $f_{\lambda_{i-2}}\left(y_{i-2}^{(i)}\right)=y_{i-1}^{(i)}$ and so

$$
\begin{array}{r}
d\left(f_{\lambda_{i-2}}\left(x_{i-3}\right), y_{i-2}^{(i)}\right) \leq d\left(f_{\lambda_{i-3}}\left(x_{i-3}\right), x_{i-2}\right)+d\left(x_{i-2}, y_{i-2}^{(i)}\right) \\
<\epsilon+\alpha_{2} \frac{\epsilon}{\alpha}<\alpha_{3} \epsilon<\epsilon L .
\end{array}
$$

Because of Lemma 1 and Corollary 1 there exists $y_{i-3}^{(i)} \in B_{\alpha_{3} \frac{\epsilon}{\alpha}}\left(x_{i-3}\right)$ such that $f_{\lambda_{i-3}}\left(y_{i-3}^{(i)}\right)=y_{i-2}^{(i)}$. Thus $d\left(f_{\lambda_{i-3}}\left(x_{i-4}\right), y_{i-3}^{(i)}\right)<\alpha_{4} \epsilon<\epsilon L$.

Repeating the process, we can find:

$y_{0}^{(i)} \in B_{\alpha_{i} \frac{\epsilon}{\alpha}}\left(x_{0}\right)$ such that $f_{\lambda_{0}}\left(y_{0}^{(i)}\right)=y_{1}^{(i)}$,

$y_{-1}^{(i)} \in B_{\alpha_{i+1} \frac{\epsilon}{\alpha}}\left(x_{0}\right)$ such that $f_{\lambda_{-1}}\left(y_{-1}^{(i)}\right)=y_{0}^{(i)}$,

$\dot{y}_{-i}^{(i)} \in B_{\alpha_{2 i} \frac{\epsilon}{\alpha}}\left(x_{0}\right)$ such that $f_{\lambda_{-i}}\left(y_{-i}^{(i)}\right)=y_{-i+1}^{(i)}$.

Since $X$ is compact, if we let $y_{k}=\lim _{i \rightarrow \infty} y_{k}^{(i)}$, then $f_{\lambda_{k}}\left(y_{k}\right)=y_{k+1}$ and $d\left(y_{k}, x_{k}\right)$ $<\epsilon L$, for all $k \in \mathbb{Z}$. Therefore $\mathcal{F}$ has the Lipschitz shadowing property.

$(i i \Rightarrow i)$. Since $\mathcal{F}$ has the shadowing property, there exist $0<\delta<\frac{\delta_{0}}{2}$ such that every $\delta \alpha$-pseudo orbit of $\mathcal{F}$ is $\delta_{0}$-shadowed by some point. Now, fix $v \in \Lambda$. Consider $x, y \in X$ such that $d\left(f_{\mathcal{v}}(x), y\right)<\delta \alpha$ and define a $\delta \alpha$-pseudo orbit of $\mathcal{F}$ by $x_{0}=x$ and $x_{i}=f_{v}^{i-1}(y)(i \in \mathbb{Z})$. Then there exists $z \in X$ and $\sigma=\left\{\ldots, \lambda_{-1}, \lambda_{0}, \lambda_{1}, \ldots\right\} \in$ $\Lambda^{\mathbb{Z}}$ such that $d\left(\mathcal{F}_{\sigma_{i}}(z), x_{i}\right)<\delta_{0}$, for all

$i \in \mathbb{Z}$. Less of generality; by proof of Theorem 2.2. in [9] and this fact that $x_{i+1}=$ $f_{v}\left(x_{i}\right)(i \in \mathbb{Z})$, we can assume that that $\lambda_{i}=v$ for all $i \geq 0$. Then $\alpha^{i-1} d\left(f_{v}(z), y\right) \leq$ $d\left(f_{v}^{i}(z), f_{v}^{i-1}(y)\right) \leq \delta_{0}$ for all $i \geq 0$, so $f_{v}(z)=y$. This implies that $z=f_{v}^{-1}(y)$ 
and $d(x, z)<\delta_{0}$, then

$d(x, z)<\frac{d\left(f_{v}(x), f_{v}(z)\right)}{\alpha}=\frac{\left(f_{v}(x), y\right)}{\alpha}<\frac{\delta \alpha}{\alpha}$. Hence $B_{\delta}(x) \cap f_{v}^{-1}(y) \neq \varnothing$. So, by Lemma $1 \mathcal{F}$ is an open IFS.

The next theorem is one of the main results of this paper and demonstrates that for an expansive IFS, the limit shadowing property and the shadowing property are equivalent.

Theorem 4. Let $X$ be a compact metric space and $\mathcal{F}=\left\{X ; f_{\lambda} \mid \lambda \in \Lambda\right\}$ be an expansive IFS on $\mathbb{Z}$. The following conditions are equivalent:

i) $\mathcal{F}$ has the shadowing property,

ii) there is a compatible metric $D$ for $X$ such that $\mathcal{F}$ has the limit shadowing property with respect to $D$.

Proof. By definitions the assertion $(i i \Rightarrow i)$ is clear.

To prove $(i \Rightarrow i i)$, at first we have the following lemmas.

Lemma 2. There is a compatible metric $D$ on $X$ and $K \geq 1$ such that

$$
\left\{\begin{array}{c}
D\left(f_{\lambda}(x), f_{\lambda}(y)\right) \leq K D(x, y) \\
D\left(f_{\lambda}^{-1}(x), f_{\lambda}^{-1}(y)\right) \leq K D(x, y)
\end{array}\right.
$$

for any $x, y \in X$ and $\lambda \in \Lambda$.

Proof. Since $\mathcal{F}$ is expansive then $f_{\lambda}$ is expansive, for every $\lambda \in \Lambda$. So, by [10] (page 3) for every $\lambda \in \Lambda$ there exists $K_{\lambda}>1$ such that

$$
\left\{\begin{array}{c}
D\left(f_{\lambda}(x), f_{\lambda}(y)\right) \leq K_{\lambda} D(x, y) \\
D\left(f_{\lambda}^{-1}(x), f_{\lambda}^{-1}(y)\right) \leq K_{\lambda} D(x, y)
\end{array}\right.
$$

for any $x, y \in X$. Take $K=\max \left\{K_{\lambda}: \lambda \in \Lambda\right\}$, the proof is complete.

To prove $(i \Rightarrow i i)$ we need to define the local stable set and the local unstable set for an IFS.

Let $\epsilon>0, \sigma \in \Lambda^{\mathbb{Z}}$ and $x$ be an arbitrary point of $X$ then

$W_{\epsilon_{0}}^{s}(x, \sigma)=\left\{y ; d\left(\widetilde{F}_{\sigma_{n}}(x), \widetilde{F}_{\sigma_{n}}(y)\right) \leq \epsilon, \forall n \geq 0\right\}$,

$W_{\epsilon_{0}}^{u}(x, \sigma)=\left\{y ; d\left(\widetilde{F}_{\sigma_{-n}}(x), \widetilde{F}_{\sigma_{-n}}(y)\right) \leq \epsilon, \forall n>0\right\}$

is said to be the local stable set and the local unstable set of $x$ respect to $\sigma \in \Lambda^{\mathbb{Z}}$.

Lemma 3. There exist constants $\epsilon_{0}>0$ and $\eta<1$ such that

$$
\left\{\begin{array}{cl}
D\left(\widetilde{F}_{\sigma_{i}}(x), \mathcal{F}_{\sigma_{i}}(y)\right) \leq \eta^{i} D(x, y) & \text { if } y \in W_{\epsilon_{0}}^{s}(x, \sigma), \\
D\left(\mathscr{F}_{\sigma_{-i}}(x), \mathcal{F}_{\sigma_{-i}}(y)\right) \leq \eta^{i} D(x, y) & \text { if } y \in W_{\epsilon_{0}}^{u}(x, \sigma)
\end{array}\right.
$$

Proof. Since for every $\lambda \in \Lambda, f_{\lambda}$ is an expansive map, To proof the lemma this is sufficient to in Lemma 1 of [13] we assume that

$$
W_{n}=\left\{(x, y) \in X \times X: d\left(\mathcal{F}_{\sigma_{i}}(x), \mathcal{F}_{\sigma_{i}}(y)\right) \leq c, \text { for all }|i|<n\right\} .
$$

The rest of proof is similar to [13]. 
( $i \Rightarrow i i$ ) Let $D$ be the compatible metric for $X$ by the above lemmas.

First, notice that if $\left\{z_{i}\right\}_{i \in \mathbb{Z}}$ is an $\epsilon-$ pseudo orbit of $\mathcal{F},\left(\epsilon \leq \frac{\epsilon_{0}}{2 L}\right)$ i.e.

$D\left(f_{\lambda_{i}}\left(z_{i}\right), z_{i+1}\right)<\epsilon$, for some $\sigma=\left\{\ldots, \lambda_{-1}, \lambda_{0}, \lambda_{1}, \ldots\right\} \in \Lambda^{\mathbb{Z}}$. Then by Theorem 3 , there exists $y \in X$ such that $D\left(\mathcal{F}_{\sigma_{i}}(y), z_{i}\right)<L \epsilon$ for all $i \in \mathbb{Z}$.

Now, suppose that $\left\{x_{i}\right\}_{i \in \mathbb{Z}}$ is a sequence of points in $X$ such that

$\lim _{i \rightarrow \pm \infty} D\left(f_{\lambda_{i}}\left(x_{i}\right), x_{i+1}\right)=0$, for some $\sigma=\left\{\ldots, \lambda_{-1}, \lambda_{0}, \lambda_{1}, \ldots\right\} \in \Lambda^{\mathbb{Z}}$. For any $\delta>0\left(\delta<\frac{\epsilon_{0}}{2 L}\right)$, there exists $I_{\delta}>0$ such that $|i|>I_{\delta}$ implies that $D\left(f_{\lambda_{i}}\left(x_{i}\right), x_{i+1}\right)<$ $\delta$. Note that

$$
\left\{\ldots, f_{\lambda_{I^{-}}-2}^{-1}\left(f_{\lambda_{I^{-1}}}^{-1}\left(x_{I_{\delta}}\right)\right), f_{\lambda_{I^{-1}}}^{-1}\left(x_{I_{\delta}}\right), x_{I_{\delta}}, x_{I_{\delta}+1}, \ldots\right\}
$$

is a $\delta$-pseudo orbit of $\mathcal{F}$, and by Theorem 3 there exists $y_{\delta} \in X$ such that $D\left(\mathcal{F}_{\sigma_{i}}\left(y_{\delta}\right), x_{i}\right)<L \delta$ for all $i \geq I_{\delta}$. By the same way, there exists $z_{\delta} \in X$, Such that $D\left(\mathcal{F}_{\sigma_{-i}}\left(z_{\delta}\right), x_{-i}\right)<L \delta$ for all $i \geq I_{\delta}$. Thus:

$$
D\left(\mathcal{F}_{\sigma_{i}}(y), \mathcal{F}_{\sigma_{i}}\left(y_{\delta}\right)\right) \leq D\left(\mathcal{F}_{\sigma_{i}}(y), x_{i}\right)+D\left(x_{i}, \mathcal{F}_{\sigma_{i}}\left(y_{\delta}\right)\right)<\epsilon_{0}
$$

for all $i \geq I_{\delta}$. This implies that $\mathcal{F}_{\sigma_{I_{\delta}}}\left(y_{\delta}\right) \in W_{\epsilon_{0}}^{s}\left(\mathcal{F}_{\sigma_{I_{\delta}}}(y)\right)$. So that, by Lemma 3, $D\left(\mathcal{F}_{\sigma_{i}}\left(y_{\delta}\right), \mathcal{F}_{\sigma_{i}}(y)\right) \leq \eta^{i-I_{\delta}} D\left(\mathcal{F}_{\sigma_{I_{\delta}}}\left(y_{\delta}\right), \mathcal{F}_{\sigma_{I_{\delta}}}(y)\right)$ for all $i \geq I_{\delta}$. Mimicking the procedure, we have $D\left(\mathcal{F}_{\sigma_{-i}}\left(y_{\delta}\right), \mathcal{F}_{\sigma_{-i}}(y)\right) \leq \eta^{i-I_{\delta}} D\left(\mathcal{F}_{\sigma_{-I_{\delta}}}\left(y_{\delta}\right), \mathcal{F}_{\sigma_{-I_{\delta}}}(y)\right)$ for all $i \geq I_{\delta}$. Take $J_{\delta}>I_{\delta}$ such that $\epsilon_{0} \eta^{i-I_{\delta}}<\delta$ if $i \geq J_{\delta}$. Since

$$
D\left(\mathcal{F}_{\sigma_{i}}(y), x_{i}\right) \leq D\left(\mathcal{F}_{\sigma_{i}}(y), \mathcal{F}_{\sigma_{i}}\left(y_{\delta}\right)\right)+D\left(\mathcal{F}_{\sigma_{i}}\left(y_{\delta}\right), x_{i}\right)
$$

and

$$
D\left(\widetilde{F}_{\sigma_{-i}}(y), x_{-i}\right) \leq D\left(\widetilde{F}_{\sigma_{-i}}(y), \widetilde{F}_{\sigma_{-i}}\left(y_{\delta}\right)\right)+D\left(\widetilde{F}_{\sigma_{-i}}\left(y_{\delta}\right), x_{-i}\right) .
$$

It is easy to see that $\max \left\{D\left(\mathcal{F}_{\sigma_{i}}(y), x_{i}\right), D\left(\mathcal{F}_{\sigma_{-i}}(y), x_{-i}\right)\right\}<(L+1) \delta$. Thus $\lim _{i \rightarrow \pm \infty} D\left(\mathcal{F}_{\sigma_{i}}(y), x_{i}\right)=0$.

Fix $\delta>0$ and $\lambda \in \Lambda$. Suppose that $\left\{x_{i}\right\}_{i \in \mathbb{Z}_{+}}$is a $\delta$-pseudo orbit for $\mathcal{F}$ and consider $\left\{y_{i}\right\}_{i \in \mathbb{Z}}$ as the following:

$$
y_{i}=\left\{\begin{array}{cc}
x_{i} & \text { if } i \geq 0, \\
f_{\lambda}^{i}\left(x_{0}\right) & \text { if } i<0 .
\end{array}\right.
$$

So, $\left\{y_{i}\right\}_{i \in \mathbb{Z}}$ is a $\delta$-pseudo orbit for $\mathcal{F}$. Then shadowing properties on $\mathbb{Z}$ implies the shadowing properties on $\mathbb{Z}_{+}$.

By Theorems 3, 4 and Theorem 3.2. of [6] we have the following corollary.

Corollary 2. Let $X$ be a compact metric space. If $\mathcal{F}=\left\{X ; f_{\lambda} \mid \lambda \in \Lambda\right\}$ is an expansive IFS with the limit (Lipschitz) shadowing property ( on $\left.\mathbb{Z}_{+}\right)$, then so is $\mathcal{F}^{-1}=\left\{X ; g_{\lambda} \mid \lambda \in \Lambda\right\}$ where $f_{\lambda}: X \rightarrow X$ is homeomorphism and $g_{\lambda}=f_{\lambda}^{-1}$ for all $\lambda \in \Lambda$.

By Theorems 3, 4 and Theorem 3.5. of [6] we have the following corollary. 
Corollary 3. Let $\Lambda$ be a finite set, $\mathcal{F}=\left\{X ; f_{\lambda} \mid \lambda \in \Lambda\right\}$ is an IFS and let $k>0$ be an integer. Set $\mathscr{F}^{k}=\left\{g_{\mu} \mid \mu \in \Pi\right\}=\left\{f_{\lambda_{k}} o \ldots o f_{\lambda_{1}} \mid \lambda_{1}, \ldots, \lambda_{k} \in \Lambda\right\}$.

If $\mathscr{F}$ has the limit (Lipschitz) shadowing property ( on $\mathbb{Z}_{+}$), then so does $\mathcal{F}^{k}$.

We say that $\mathcal{F}$ is strongly expansive if there exist a metric $d$ for $X$ and a constant $e \geq 0$ such that for every two arbitrary $\sigma, \mu \in \Lambda^{\mathbb{Z}}$,

$d\left(\mathcal{F}_{\sigma_{i}}(x), \mathcal{F}_{\mu_{i}}(y)\right)<e$, for all $i \in \mathbb{Z}$, implies that $x=y$.

To prove Theorem 5 , we need the following lemma.

Lemma 4. Suppose that $\mathcal{F}$ is an expansive IFS with expansive constant $e, \alpha$ is an arbitrary positive number and $\sigma, \mu \in \Lambda^{\mathbb{Z}}$. For all $x, y \in X$ there exists an integer $N=N(e, \alpha)>0$ such that if $d\left(\widetilde{F}_{\sigma_{i}}(x), \mathcal{F}_{\mu}(y)\right) \leq e$ for all $|i| \leq N$, then $d(x, y)<$ $\alpha$.

Proof. We will give a proof by contradiction. Suppose that for each $n \geq 1$, there exist $x_{n}$ and $y_{n}$ with $d\left(\mathcal{F}_{\sigma_{i}}\left(x_{n}\right), \mathcal{F}_{\mu_{i}}\left(y_{n}\right)\right) \leq e$ for all $|i| \leq n$ and $d\left(x_{n}, y_{n}\right) \geq \alpha$. Since $X$ is a compact metric space, we may assume that $x_{n} \rightarrow x$ and $y_{n} \rightarrow y$ and hence that $\left.\mathscr{F}_{\sigma_{i}}\left(x_{n}\right) \rightarrow \mathcal{F}_{\sigma_{i}}(x) \mathcal{F}_{\mu_{i}}\left(y_{n}\right)\right) \rightarrow \mathscr{F}_{\mu}(y)$ for every $|i| \geq 1$. Then $d\left(\widetilde{F}_{\sigma_{i}}(x), \mathcal{F}_{\mu_{i}}(y)\right) \leq e$ for all $i \in \mathbb{Z}$ and $d(x, y) \geq \alpha$, which is a contradiction.

Theorem 5. Let $X$ be a compact metric space and $\mathcal{F}=\left\{X ; f_{\lambda} \mid \lambda \in \Lambda\right\}$ be an strongly expansive IFS on $\mathbb{Z}$. The following conditions are equivalent:

i) $\mathcal{F}$ has the shadowing property,

ii) $\mathscr{F}$ has the continuous shadowing property.

Proof. By definitions, this is clear that continuous shadowing property implies the shadowing property.

$(i \Rightarrow i i)$. Let $e>0$ be an expansive constant of $\mathcal{F}$. For $0<\epsilon<\frac{e}{3}$, let $\delta=\delta(\epsilon)<$ $\epsilon$ be as in the shadowing property of $\mathcal{F}$. It is easy to see that for any $\delta$-pseudo orbit $\left\{x_{i}\right\}_{i \in \mathbb{Z}}$ of $\mathcal{F}$ by Remark 2 there exists $\sigma=\left\{\ldots, \lambda_{-1}, \lambda_{0}, \lambda_{1}, \ldots\right\} \in \Lambda^{\mathbb{Z}}$ and a unique $y \in X$ satisfying $d\left(\mathcal{F}_{\sigma_{i}}(y), x_{i}\right)<\epsilon$ for all $i \in \mathbb{Z}$. So we define $r: P(\mathcal{F}, \delta) \rightarrow$ $X$ by $r\left(\left\{x_{i}\right\}_{i \in \mathbb{Z}}\right)=y$. To show that the map $r$ is continuous, choose an arbitrary constant $\alpha>0$, by Lemma 4 there exists an integer $N=N(e, \alpha)>0$ such that if $d\left(\mathcal{F}_{\sigma_{i}}(y), \mathcal{F}_{\mu_{i}}\left(y^{\prime}\right)\right) \leq e$ for $\sigma, \mu \in \Lambda^{\mathbb{Z}}$ and all $|i| \leq N$, then $d\left(y, y^{\prime}\right)<\alpha$. Pick $\beta>0$ such that $2^{N} \beta<\frac{e}{3}$. Let $\left\{x_{i}\right\}_{i \in \mathbb{Z}},\left\{x_{i}^{\prime}\right\}_{i \in \mathbb{Z}} \in P(\mathcal{F}, \delta)$ be given two $\delta$-pseudo orbit of $\mathcal{F}$ with related sequences $\sigma, \mu \in \Lambda^{\mathbb{Z}}$ and let $r\left(\left\{x_{i}\right\}_{i \in \mathbb{Z}}\right)=y$ and $r\left(\left\{x_{i}^{\prime}\right\}_{i \in \mathbb{Z}}\right)=y^{\prime}$. if $d\left(\left\{x_{i}\right\}_{i \in \mathbb{Z}},\left\{x_{i}^{\prime}\right\}_{i \in \mathbb{Z}}\right)<\beta$, then we see that $d\left(x_{i}, x_{i}^{\prime}\right)<\frac{e}{2}$ for all $|i|<N$, so that $d\left(\mathcal{F}_{\sigma_{i}}(y), \mathcal{F}_{\mu_{i}}\left(y^{\prime}\right)\right) \leq d\left(\mathcal{F}_{\sigma_{i}}(y), x_{i}\right)+d\left(x_{i}, x_{i}^{\prime}\right)+d\left(\mathcal{F}_{\mu_{i}}\left(y^{\prime}\right), x_{i}^{\prime}\right)<e$ for all $|i| \leq N$. Thus $d\left(y, y^{\prime}\right)<\alpha$ by the choice of $N$, and the conclusion is obtained. 


\section{REFERENCES}

[1] N. Aoki and K. Hiraide, Topological theory of dynamical systems, 1st ed., ser. Series is books. North-Holland: North-Holland Mathematical Library, 1994, vol. 52.

[2] M. Barnsley, Fractals everywhere. Boston: Academic Press, 1988.

[3] M. Barnsley and A. Vince, "Fractal continuation of analytic (fractal) functions," ArXiv:1209.6100v1.

[4] M. Barnsley and A. Vince, "The conley attractor of an iterated function system," Bull. Aust. Math. Soc., vol. 88, no. 3, pp. 267-279, 2013.

[5] E. Coven and W. Reddy, "Positively expansive maps of compact manifolds," in Lecture Notes in Math., ser. Internat. Conf., Northwestern Univ., Evanston, Ill., 1979, vol. 819. Berlin: Springer, 1980, pp. 96-110.

[6] M. Fatehi Nia, "Iterated function systems with the shadowing property," J. Adv. Res. Pure Math., vol. 7, no. 1, pp. 83-91, 2015.

[7] M. Fatehi Nia, "Parameterized ifs with the asymptotic average shadowing property," Qual. Theory Dyn. Syst., vol. 15, no. 2, pp. 367-381, 2016.

[8] M. Fatehi Nia, "Adding machine maps and minimal sets for iterated function systems." J. Dyn. Syst. Geom. Theor, vol. 15, no. 1, pp. 71-83, 2017.

[9] V. Glavan and V. Gutu, "Shadowing in parameterized ifs," Fixed Point Theory, vol. 7, pp. 263-274, 2006.

[10] S. K., "Various shadowing properties for positively expansive maps," Topology. Appl., vol. 131, pp. $15-31,2003$.

[11] K. Lee and K. Sakai, "Structural stability of vector fields with shadowing," J. Differential Equations., vol. 232, pp. 303-313, 2007.

[12] K. Palmer, Shadowing in dynamical systems. Theory and applications. Dordrecht: Kluwer Academic Publishers, 2000.

[13] W. Reddy, "Expansive canonical coordinates are hyperbolic," Topology Appl., vol. 15, pp. 205210, 1983.

[14] K. Sakai and K. Lee, "Various shadowing properties and their equivalence," Topology. Appl., vol. 13, pp. 533-539, 2005.

Author's address

\section{Mehdi Fatehi Nia}

Yazd University, Department of Mathematics, 89195-741 Yazd, Iran

E-mail address: fatehiniameyazd.ac.ir 